\title{
Captain Herbert B. Tanowitz, M.D., F.I.D.S.A., F.A.C.P., F.R.S.T.M.H., 1941-2018
}

It is with a heavy heart that we report the passing of Dr. Herbert Tanowitz on July 17th, 2018.

Dr. Tanowitz received his medical degree from the Albert Einstein College of Medicine in 1967, where he was inducted into the Alpha Omega Alpha Medical Honor Society. After an internship at Bellevue Hospital in New York (Figure 1), followed by an Internal Medicine residency at Lincoln Hospital, an affiliate of Albert Einstein College of Medicine, he returned to Einstein for an Infectious Diseases fellowship, after which he briefly remained on the faculty before joining the Naval Reserves as a medical officer in 1973. Herb served as Assistant Chief of Medicine at the Naval Hospital in Quantico, VA, until 1975, when he returned to Einstein as Assistant Professor of Pathology and Medicine; however, he continued to serve and rose through the ranks to become a captain in the Medical Corps until his retirement from the United States Navy Reserve in 2008. Even after retirement, it was not unusual for Herb to make the trip from his home in Westchester County to the Armed Forces Reserve Center in Farmingdale, Long Island, to "help out from time to time" on weekends. Upon returning to Einstein, Herb served as an Attending Physician at the Jack D. Weiler Hospital and Montefiore Medical Center and at Jacobi Hospital. He also served as an Infectious Diseases Consultant at Our Lady of Mercy Medical Center. Herb was a Hospital Epidemiologist at Weiler Hospital from 1990 to 1996, and served as Director

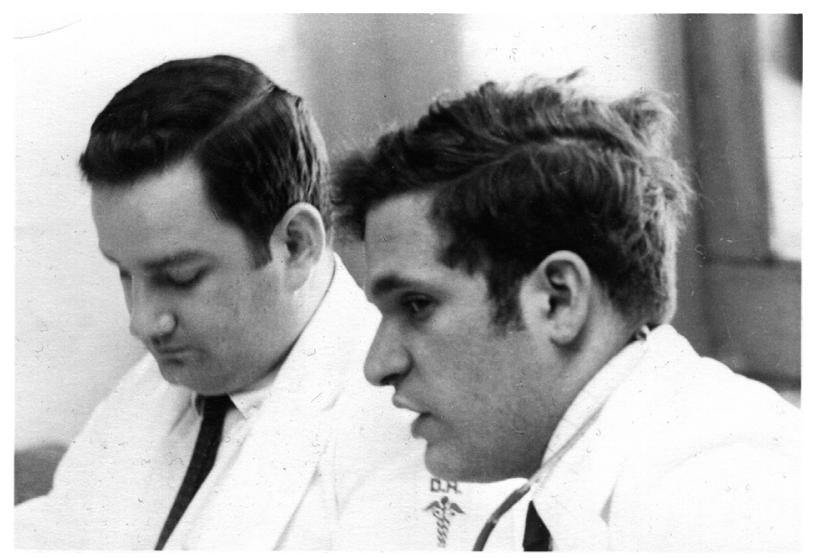

Figure 1 Dr. Herbert Tanowitz (right) during his intern year at Bellevue Hospital in 1968. Image courtesy of Dr. Gerald Friedland, M.D.

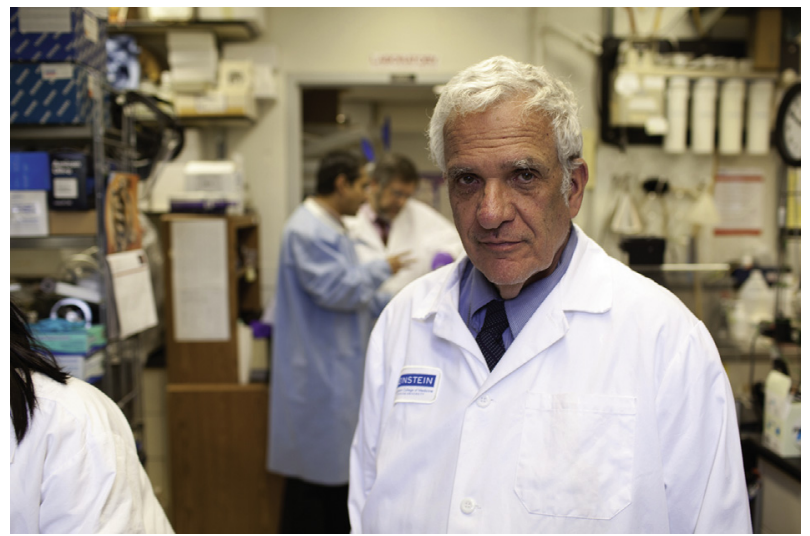

Figure 2 Dr. Herbert Tanowitz. Image courtesy of Albert Einstein College of Medicine.

of the Diagnostic Parasitology Laboratory and Associate Director of the Parasitology Clinic at Jacobi Hospital from 2010 to 2018. He was beloved by his patients as well as fellows and students who rotated with him. Herb often joked that he was the longest serving Infectious Diseases fellow of the Einstein fellowship program, because an official graduation ceremony was never held after completion of his fellowship. He remained at Einstein, until his death, as Professor of Pathology and Medicine (Figure 2). In 2010, he received the Dominick P. Purpura Distinguished Alumnus award from Einstein.

Throughout his prolific career, perhaps one of Herb's most memorable roles was as an educator and mentor. He taught in the Parasitology course at the medical school and the Microbial Pathogenesis course at the graduate school. He was also an active participant in graduate medical education, teaching medical students, residents, and fellows on medical rounds, and actively participating as a lecturer in the several didactic lecture series. Herb also served on over 30 thesis advisory committees and thesis and qualifying examination committees. Ever the consummate mentor, Herb was the Principal Investigator on multiple training grants, including the NIH Fogarty International Training Grant, with the goal of training Brazilian students and post-doctoral fellows, and bringing them to Einstein to do research in different laboratories. He was also the Director and Principal Investigator of 
the NIH T32 Training Grant in Geographic Medicine and Emerging Infections. Finally, Herb had an international commitment to nurturing young Chagas disease scientists and he provided advice and encouragement to dozens of young individuals over four decades who are now leaders in the field. The success of his trainees speaks to his dedication to fostering new generations of scientists, with former postdoctoral trainees, clinical residents and fellows, and Fogarty/ T32 training grant participants holding faculty positions at various universities or the CDC.

The bulk of Herb's research focused on Chagasic cardiomyopathy and on the mechanisms of vascular and metabolic changes induced by Trypanosoma cruzi infection. He was instrumental in pioneering the studies on the role of endothelin, the vasoactive peptide, in Chagas disease, reporting improved cardiac function with inhibition of endothelin or its receptors in murine models. Herb also discovered that T. cruzi regulates mammalian host response by endogenously synthesizing thromboxane, another vasoactive peptide. Herb demonstrated that $T$. cruzi invades mammalian adipose tissue to avoid clearance by the immune system, in essence creating a reservoir for persistent chronic infection, with unexpected deleterious outcomes for host metabolic responses. He was a dynamic researcher who published over 300 articles and over 30 book chapters. Herb was a sought-after speaker for many national, international, and regional events, and he received several accolades in recognition of his scholarship, including induction into the prestigious Sigma Xi Scientific Research Honor Society and The Brazilian Academy of Sciences.
Herb's service to the scientific community was widely recognized. He served as active member and Chair of several NIH review panels. He was also a member of the American Society for Investigative Pathology (beginning in 2005) and worked with the American Journal of Pathology, serving as Senior Associate Editor (2008 to 2012), Associate Editor (2012 to 2014), and as a member of the editorial board until his passing. Herb was also a Managing Editor for Frontiers in Bioscience from 2001 until 2018, and was a founding Co-Editor-in-Chief of the Journal of Neuroinfectious Diseases.

Herb is survived by his children Pam, Meredith, and Jill, and their families.

An honorary lecture fund has been created by the Department of Pathology at Einstein, The Dr. Herbert B. Tanowitz Honorary Lecture Fund for Parasitology and Tropical Medicine, to support an annual lecture focusing on parasitology and tropical medicine in his honor. To donate, please visit http://www.einstein.yu.edu/donors/tanowitzmemorial-lecture-fund.

Mahalia S. Desruisseaux Department of Medicine, Yale University School of Medicine, New Haven, Connecticut

David M. Engman

Department of Pathology and Laboratory Medicine, Cedars Sinai Medical Center, Los Angeles, California 